\title{
Tranverse Momentum Distribution in Quarkonium Photoproduction in pp and AA Collisions at the LHC
}

\author{
M. B. Gay Ducati*t; $\quad$ F. Kopp $\ddagger \quad$ M. V. T. Machado ${ }^{\S}$ and S. Martins \\ High Energy Physics Phonomenology Group, GFPAE IF-UFRGS \\ Caixa Postal 15051, CEP 91501-970, Porto Alegre, Rio Grande do Sul, Brazil
}

\begin{abstract}
The exclusive photoproduction of the heavy vector mesons $V(\Psi(1 S), \Psi(2 S), \Upsilon(1 S), \Upsilon(2 S))$ is investigated in the context of ultra-peripheral collisions proton-proton and nucleus-nucleus for the energies available at the LHC run 2. The rapidity distribution was calculated in order to test the robustness of the models GBW and CGC against the previous experimental results considering $\Psi(1 S), \Psi(2 S)$ and $\Upsilon(1 S)$ states. Results for the transverse momentum distribution were obtained for the $\mathrm{pp}$ and $\mathrm{PbPb}$ collisions, in the theoretical framework of the light-cone color dipole formalism, which includes consistently parton saturation effects and nuclear shadowing corrections.
\end{abstract}

38th International Conference on High Energy Physics

3-10 August 2016

Chicago, USA

\footnotetext{
* Speaker.

†beatriz.gay@ufrgs.br

$\ddagger$ fabio.kopp@ufrgs.br

$\S$ magno.machado@ufrgs.br

ฯ sony.martins@ufrgs.br
} 


\section{Introduction}

In the ultra-peripheral collisions regime [1], where the impact parameter is larger than the sum of the radius the interaction hadrons, the quarkonium hadroproduction can be neglected in comparison to the exclusive photoproduction. This one dominates the process through the emission of virtual photons that interact with the target, given rise to the photoproduction cross section. The theoretical framework considered in the analysis of this process is the light-cone color dipole formalism [2], in which the amplitude can be written as a convolution between the photon-meson wave functions overlap and the elementary dipole-target cross section [3].

In this work, it was investigate the transverse momentum distribution for the exclusive photoproduction of mesons $V(\Psi(1 S), \Psi(2 S), \Upsilon(1 S), \Upsilon(2 S))$ in proton-proton and nucleus-nucleus collisions in the LHC energy interval (Run 2) [4]. The photoproduction provides ways to investigate the transition between the linear and the non-linear dynamics where the physical process of the partonic recombination, e.g. $g g \rightarrow g$, becomes important. This transition is characterized by the limitation on the maximum phase-space parton density that can be reached in the hadron wave-function, due to the parton saturation phenomenon [5, 6], introducing a saturation scale $Q_{\text {sat }}$, which is enhanced in the nuclear case. The theoretical framework chosen includes consistently both the parton saturation effects in photon-proton interaction and the nuclear shadowing effects in photon-nucleus process.

In the next section we summarize the main theoretical information and phenomenological inputs used to compute the rapidity and transverse momentum distributions of the $V$ states in $p p$ and $A A$ collisions. In the section 3 we compare our results in rapidity with the experimental data measured by LHCb Collaboration [7] for $\Psi(1 S, 2 S)$ and $\Upsilon(1 S)$ in $p p$ collisions, concluding the section with our estimates for the transverse momentum distribution in the LHC energy (Run 2).

\section{Theoretical Framework}

In the present work, we consider the color dipole approach for modeling the photon-target interaction, in which the photoproduction cross section in pp collision, $\sigma_{\gamma}^{p}$, is given by $[8,9]$

$$
\sigma(\gamma p \rightarrow V p)\left(s, Q^{2}\right)=\frac{1}{16 \pi B_{V}}\left|\operatorname{Im} A\left(x, Q^{2}, \Delta=0\right)\right|^{2}\left(1+\beta^{2}\right) R_{g}\left(\lambda_{e f f}\right) .
$$

The parameter $B_{V}$ (slope parameter) was calculated from the Regge phenomenology [10] with the energy dependence. The parameters $\beta$ and $R_{g}$ represents, respectively, the real contribution of the frontal amplitude [11] and the skewedness effects which takes into account the off-forward features of the process (different transverse momentum of the exchanged gluons in the t-channel) [12]. Finally, the $A\left(x, Q^{2}, \Delta=0\right)$ is the forward amplitude [3] fatorized as

$$
A^{\gamma^{*} p \rightarrow V p}(x, \Delta=0)=\int d^{2} \mathbf{r} \int_{0}^{1} \frac{d z}{4 \pi} \rho_{V}(z, r) A_{q \bar{q}}(x, r, \Delta=0),
$$

where the quantity $\rho_{V}=\left(\Psi_{V}^{*} \Psi\right)_{T}$ represents the overlap of the photon-meson wave functions, found in Ref. [11], and $A_{q \bar{q}}$ is the dipole-target scattering amplitude (assumed to be imaginary). The kinematical variable $x=M_{V}^{2} /(2 \omega \sqrt{s})$ is the Bjorken variable and the squared momentum 

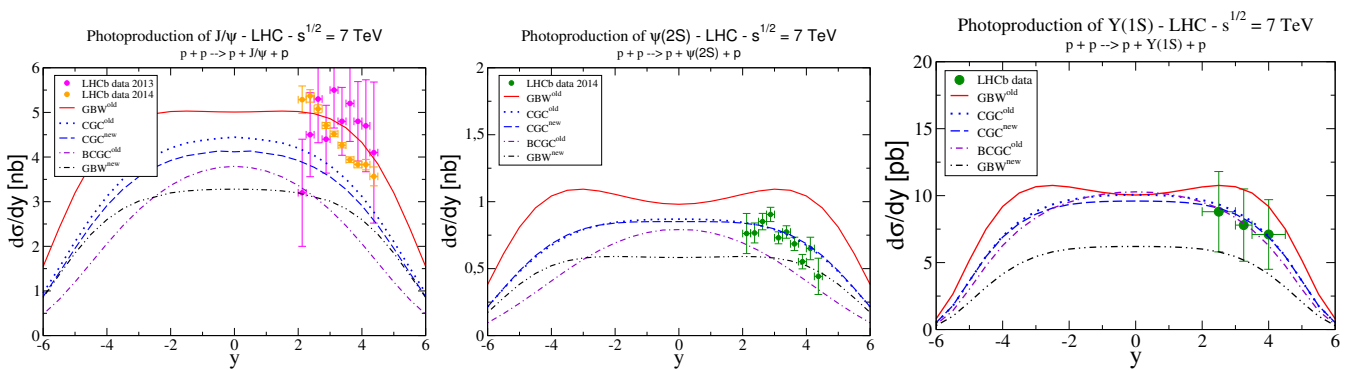

Figure 1: Rapidity distribution for the vector meson states $\Psi(1 S)$ (left panel), $\Psi(2 S)$ (central panel) and $\Upsilon(1 S)$ (right panel) in $p p$ collisions in the LHC Run I at $\sqrt{s}=7 \mathrm{TeV}$. Data from Refs. [13, 14, 15]

transfer in hadron vertex is $t=-\Delta^{2}$. The variables $z$ and $r$ are the longitudinal momentum fraction carried by the quark and the transverse color dipole size, respectively.

In the case of nuclear targets, the coherent photonuclear cross section was calculated using the following expression [16],

$$
\sigma(\gamma A \rightarrow V A)(\omega)=R_{g}^{2} \frac{\left|A_{\text {пис }}(x, \Delta=0)\right|^{2}}{16 \pi}\left(1+\beta^{2}\right) \int_{t_{\min }}^{\infty}|F(t)|^{2} d t
$$

where $t_{\min }=\left(m_{V}^{2} / 2 \omega\right)^{2}$ and the nuclear form factor is given by $F(q)=\frac{4 \pi \rho_{0}}{A q^{3}}\left[\sin \left(q R_{A}\right)-q R_{A} \cos \left(q R_{A}\right)\right]$ $\times\left[\frac{1}{1+a^{2} q^{2}}\right]$, with $q=\sqrt{|t|}, \rho_{0}=0.1385 \mathrm{fm}^{-3}$ and $a=0.7 \mathrm{fm}$.

In contrast to the photon wave-functions, which can be completely computed from perturbation theory [2], the meson wave-function carries a phenomenological input embedded in functions $\phi_{T, L}$. In our calculations, we use the Boosted-Gaussian model [11] since it can be applied in a systematic way for excited states. The parameters $\mathscr{R}_{n S}^{2}$ and $\mathscr{N}_{n S}$ presented in the model were calculated in $[17,18]$.

Other important component in Eq. (2.1) is the dipole scattering amplitude, which is related to the color dipole cross section in the form,

$$
\sigma_{q \bar{q}}(x, r)=2 \int d^{2} b A_{q \bar{q}}(x, r, b)
$$

bearing in mind that $b$ and $\Delta$ are Fourier conjugates variables.

We considered the models GBW [19] and GBW-KSX [20], the last one taking into account the effect of the gluon number fluctuations. It was also consider the CGC model [21], labeled for CGC-old parameterization [22], which considers the previous DESY-HERA data and CGC-new [23], which considers more recent data from ZEUS and H1 combined results for inclusive DIS. Finally, it was included the b-CGC model [24], with impact parameter dependence in the dipoleproton scaterring amplitude.

In the next section, we provide predictions to the future (current) runs of LHC for the rapidity and transverse momentum distributions of the $V(\Psi(1 S), \Psi(2 S), \Upsilon(1 S), \Upsilon(2 S))$ states in ultraperipheral collisions. 


\section{Results and Discussions}

In pp collisions, the rapidity distribution of the vector meson $\mathrm{V}$ is given by $[8,9]$

$$
\frac{d \sigma}{d y}(p+p \rightarrow p+p+V)=S_{\text {gap }}^{2}\left[\omega \frac{d N_{\gamma}^{p}(\omega)}{d \omega} \sigma(\gamma p \rightarrow V+p)+(y \rightarrow-y)\right]
$$

where the parameter $S_{\text {gap }}^{2}$ quantifies the absorptive corrections. Here, was taken $S_{\text {gap }}^{2}=0.8$ [25]. To test the precision of the models, it was calculated the rapidity distribution in order to compare with the available data of LHC for the $J / \psi, \Psi(2 S)$ and $\Upsilon(1 S)$ production in $\sqrt{s}=7 \mathrm{TeV}$, Figure 1.

\begin{tabular}{|c|ccccc|c|}
\hline \hline$\sigma_{p p \rightarrow J / \psi \rightarrow \mu^{+} \mu^{-}}$ & $G B W$ & $C G C^{\text {old }}$ & $C G C^{\text {new }}$ & BCGC $^{\text {old }}$ & $G B W^{k s x}$ & LHCb measure \\
\hline$\psi(1 s)$ & 277.60 & 213.69 & 199.58 & 154.57 & 170.81 & $291 \pm 20.24$ \\
$\psi(2 s)$ & 8.40 & 5.94 & 5.98 & 4.13 & 4.39 & $6.5 \pm 0.98$ \\
\hline$\Upsilon(1 s)$ & 25.05 & 20.45 & 20.02 & 19.12 & 12.5 & $9.0 \pm 2.7$ \\
$\Upsilon(2 s)$ & 4.32 & 3.8 & 3.70 & 3.9 & 2.05 & $1.3 \pm 0.85$ \\
\hline \hline
\end{tabular}

Table 1: Total cross section in the rapidity region $2.0<\eta_{\mu^{ \pm}}<4.5$ (in units of $p b$ ) for photoproduction of the $\psi(1 S, 2 S)$ (corrected for acceptance) and $\Upsilon(1 S, 2 S)$ states in $p p$ collisions at $\sqrt{s}=7 \mathrm{TeV}$ compared to the $\mathrm{LHCb}$ data.

The relative normalization and the overall behavior on rapidity are well reproduced by all the models in the forward region in comparison to the experimental results from LHCb Collaboration. However, due to the limited experimental data, we can not effectively discard the dipole models for the exclusive photoproduction of the $J / \psi$ and $\psi(2 S)$. In the $\Upsilon(1 S)$ production, all models are within experimental errors, which does not allow to make a confident discrimination among the possible choices.
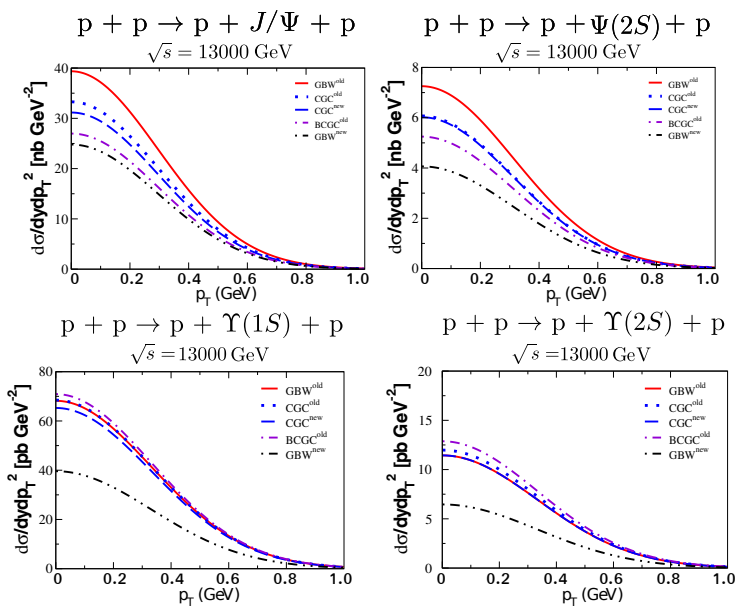

Figure 2: Results for the $p_{T}$ distribution of the $\Psi(1 S, 2 S)$ and $Y(1 S, 2 S)$ states in pp collisions at $\sqrt{s}=13$ $\mathrm{TeV}$. 
In order to complement our analysis, it was calculated the total cross section in the rapidity interval $2.0<y<4.5$. The results can been seen in the table 1. In contrast of $\psi^{\prime} s$, the results of the $\Upsilon(1 S, 2 S)$ need the experimental results of acceptance.
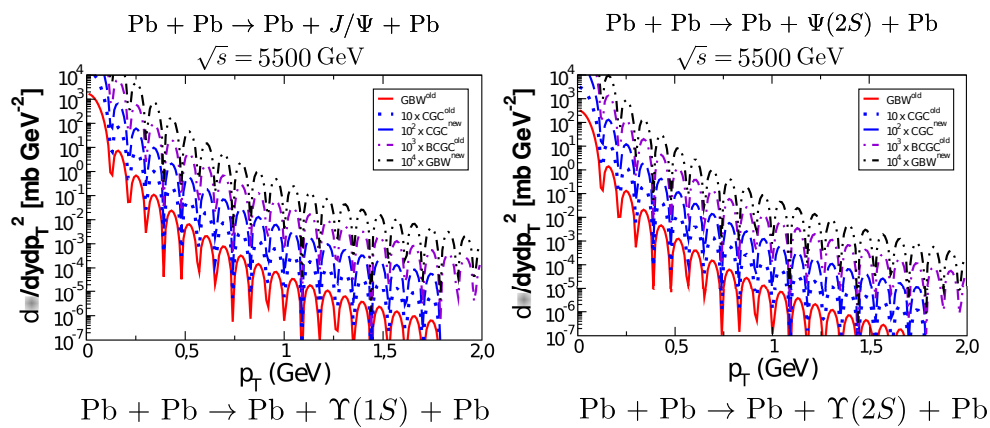

$$
\mathrm{Pb}+\mathrm{Pb} \rightarrow \mathrm{Pb}+\Upsilon(2 S)+\mathrm{Pb}
$$
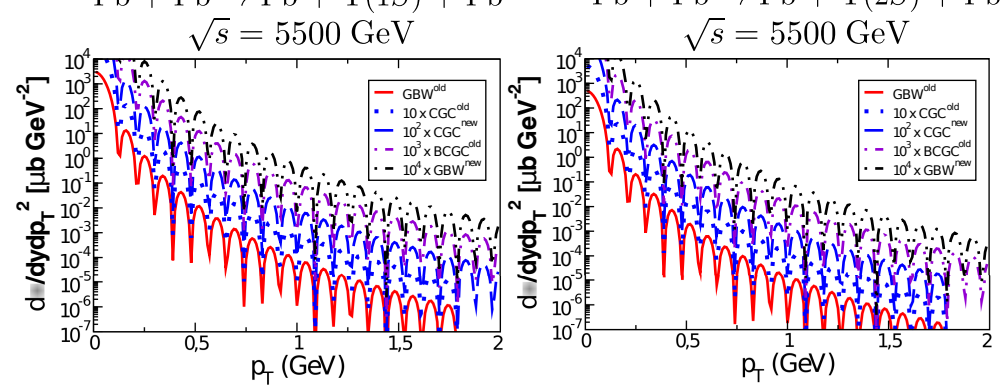

Figure 3: Results for the $p_{T}$ distribution of the $\Psi(1 S, 2 S)$ and $Y(1 S, 2 S)$ states in $\mathrm{PbPb}$ collisions at $\sqrt{s}=5.5$ $\mathrm{TeV}$.

At last, it was calculated the $p_{T}$-distribution for the vector mesons $\mathrm{V}$ in pp and AA collisions in the central rapidity region $(y=0)$. It was pointed in [26] the existence of an excess of $J / \Psi$ production at forward rapidity at very low $p_{T}\left(p_{T}<300 \mathrm{MeV} / \mathrm{c}\right)$, which could be caused by photoproduction in the peripheral regime. Thus, as a first step towards this regime, we calculated the $p_{T}$-distribution in the ultra-peripheral collisions using the results obtained for the central rapidity distribution. In pp collision case, the $p_{T}$-distribution is given, approximately, by:

$$
\left.\left.\frac{d^{2} \sigma}{d y d p_{T}^{2}}\right|_{y=0} \approx \frac{d \sigma}{d y}\right|_{y=0} B_{V}(y=0) e^{-B_{V} p_{T}^{2}}
$$

From eq. (3.2), in Fig. 2 we presented our estimates for pp collisions corresponding to the production of the $\Psi(1 S, 2 S)$ and $\Upsilon(1 S, 2 S)$ at $\sqrt{s}=13 \mathrm{TeV}$. It was obtained the Gaussian behavior consistent with the exponencial ansatz, $A_{q q} \propto \mathrm{e}^{-B_{V} \Delta^{2} / 2}$, applied in the DESY-HERA experimental data on vector meson photoproduction.

On the other hand, for the $\mathrm{PbPb}$ collision, the momentum transverse distribution in the central region $(y=0)$ is given by

$$
\left.\frac{d^{2} \sigma}{d y d p_{T}^{2}}\right|_{y=0}=\frac{\left.\frac{d \sigma}{d y}\right|_{y=0}\left|F\left(|t|=p_{T}^{2}\right)\right|^{2}}{\int_{-\infty}^{t_{\min }}\left|F\left(|t|=p_{T}^{2}\right)\right|^{2} d t} \quad \text { with } \quad t_{\min }=\left(\frac{m_{V}^{2}}{4 \omega}\right)^{2}
$$

In this case, it were obtained the results of the Fig. 3, where the form factor used gives rise to oscillatory behavior that is a characteristic of diffractive processes. 


\section{References}

[1] G. Baur, K. Hencken, D. Trautmann, S. Sadovsky, Y. Kharlov, Phys. Rep. 364, 359 (2002); C. A. Bertulani, S. R. Klein and J. Nystrand, Ann. Rev. Nucl. Part. Sci. 55, 271 (2005).

[2] N. N. Nikolaev, B. G. Zakharov, Phys. Lett. B 332, 184 (1994); Z. Phys. C 64, 631 (1994).

[3] J. Nemchik, N. N. Nikolaev, E. Predazzi and B. G. Zakharov, Phys. Lett. B 374, 199 (1996).

[4] M. B. Gay Ducati, F. Kopp, M. V. T. Machado and S. Martins, Phys. Rev. D 94, 094023 (2016).

[5] F. Gelis, E. Iancu, J. Jalilian-Marian and R. Venugopalan, Ann. Rev. Nucl. Part. Sci. 60, 463 (2010); H. Weigert, Prog. Part. Nucl. Phys. 55, 461 (2005); J. Jalilian-Marian and Y. V. Kovchegov, Prog. Part. Nucl. Phys. 56, 104 (2006).

[6] M.B. Gay Ducati, Braz. J. Phys., v. 31, 115 (2001); A. Ayala, M.B. Gay Ducati and E.M. Levin, Eur. Phys. J. C 8, 115 (1999); A. Ayala, M.B. Gay Ducati and E.M. Levin, Phys. Lett. B 388, 188 (1996); A. Ayala, M.B. Gay Ducati and E.M. Levin, Nucl. Phys. B 493, 305 (1997); A. Ayala, M.B. Gay Ducati and E.M. Levin, Nucl. Phys. B 511, 355 (1998).

[7] R. Aaij et al. [LHCb Collaboration], JHEP 1509, 084 (2015).

[8] M. B. Gay Ducati, M.T. Griep, M.V.T. Machado, Phys. Rev. D 88, 017504 (2013).

[9] M.B. Gay Ducati, M.T. Griep, M.V.T. Machado, Phys. Rev. C 88, 014910 (2013).

[10] G. S. Santos and M. V. T. Machado, J. Phys. G 42, 105001 (2015)

[11] J. Nemchik, N. N. Nikolaev, E. Predazzi, and B. G. Zakharov, Z. Phys. C 75, 71 (1997).

[12] A. G. Shuvaev, K. J. Golec-Biernat, A. D. Martin, and M. G. Ryskin, Phys. Rev. D 60, 014015 (1999).

[13] R. Aaij et al. [LHCb Collaboration], J. Phys. G 40, 045001 (2013).

[14] R. Aaij et al. [LHCb Collaboration], J. Phys. G 41, 055002 (2014).

[15] R. Aaij et al. [LHCb Collaboration], JHEP 1509, 084 (2015).

[16] S. Klein and J. Nystrand, Phys. Rev. C 60, 014903 (1999).

[17] N. Armesto and A. H. Rezaeian, Phys. Rev. D 90, 054003 (2014).

[18] B.E. Cox, J. R. Forshaw and R. Sandapen, JHEP 0906, 034 (2009).

[19] K. Golec-Biernat and M. Wüsthoff, Phys. Rev. D 59, 014017 (1998).

[20] M. Kozlov, A. Shoshi and W. Xiang, JHEP 0710, 020 (2007).

[21] E. Iancu, K. Itakura, S. Munier, Phys. Lett. B 590, 199 (2004).

[22] G. Soyez, Phys. Lett. B 655, 32 (2007).

[23] A. H. Rezaeain and I. Schmidt, Phys. Rev. D 88, 074016 (2013).

[24] H. Kowalski, L. Motyka and G. Watt, Phys. Rev. D 74, 074016 (2006)

[25] W. Schafer and A. Szczurek, Phys. Rev. D 76, 094014 (2007).

[26] J. Adam et al. [ALICE Collaboration], Phys. Rev. Lett. 116, 222301 (2016). 